\title{
Starting at the Roots: Using Human-Centered Design to Develop a Sex and Pregnancy Education Curriculum for Adolescents in Kenya
}

\author{
Sean Buehler, BSPH ${ }^{1}$, Julie Thorne, MD, $\mathrm{MPH}^{2}$ \\ ${ }^{1}$ Indiana University School of Medicine, ${ }^{2}$ University of Toronto School of \\ Medicine, Faculty of Medicine
}

\begin{abstract}
:
The rate of adolescent pregnancy worldwide remains unacceptably high. Sixteen million adolescent girls between the ages of 15 and 19, and 2 million under the age of 15 become pregnant each year. Ninety-five percent of these births occur in low-income countries, with four times the rate of adolescent pregnancy in the poorest regions of the world compared to high-income countries. There has been a shift globally to focus on the sexual and reproductive health needs of adolescents, including adolescent pregnancy. With increased awareness of this need has come a renewed call for evidence-based provision of adolescentfocused sexual and reproductive health (ASRH) services, as well as programs to prevent pregnancy in this age group.

"Human-centered design" methodology is emerging as an innovative, feasible, and effective participatory approach to program design and implementation in health care. The standard Human Centered Design format includes 5 steps: (1) Understand people's experiences, challenges, and priorities; (2) Use existing knowledge and new research to define and clarify the problem; (3) Prompt creative thinking to design many different solutions; (4) Build and workshop prototypes of ideas to quickly learn how they can be improved; and (5) Deliver solutions that meet the needs of the target population.

Through this process, design team members identified a need to "help adolescents by removing the uncertainty that surrounds information on pregnancy and treatment choices". To meet this need, we utilized participatory program development to build an adolescent-specific sex and pregnancy education program at Moi Teaching and Referral Hospital in Eldoret, Kenya.
\end{abstract}

